\title{
SARS-CoV-2 Infection Elicits A Greater Humoral Immune Response In The Second Wave Than The First Wave In Morocco
}

\section{Sayeh Ezzikouri ( $\square$ sayeh.ezzikouri@pasteur.ma )}

Institut Pasteur du Maroc

Hind Majidi

Ministry of Health of Morocco

Soad Redwane

Direction Régionale de la santé Casablanca-Settat, Observatoire régional de santé, Casablanca

Nadia Meziane

Centre Régional de Transfusion Sanguine, Casablanca

Abdellatif Zahir

Centre Transfusion Sanguine, El Jadida

Islam Abbadi

Institut Pasteur du Maroc

Chaimaa Zerrad

Institut Pasteur du Maroc

Asmaa Haddaji

Institut Pasteur du Maroc

Karima Abounouh

Institut Pasteur du Maroc

Soufiane Hilmi

Institut Pasteur du Maroc

Safaa Aqillouch

Institut Pasteur du Maroc

Mohcine Elmessaoudi-Idrissi

Institut Pasteur du Maroc

Oumaima Laazaazia

Institut Pasteur du Maroc

Abdelhakim Ainahi

Institut Pasteur du Maroc

Nabila Rmili

Direction Régionale de la santé Casablanca-Settat, Casablanca

Abderrahmane Maaroufi 
Institut Pasteur du Maroc

Soumaya Benjelloun

Institut Pasteur du Maroc

\section{Research Article}

Keywords: COVID-19, SARS-CoV-2, antibody, B cells, vaccine

Posted Date: May 27th, 2021

DOl: https://doi.org/10.21203/rs.3.rs-518276/v1

License: (1) This work is licensed under a Creative Commons Attribution 4.0 International License. Read Full License 


\section{Abstract}

Purpose: Severe acute respiratory syndrome coronavirus 2 (SARS-CoV-2) is the causative agent of the ongoing pandemic of coronavirus disease 2019 (COVID-19). One year has passed since the first COVID19 case, and concerns have emerged regarding new variants, meaning that characterizing antibodymediated immune response dynamics is of paramount importance. Here, we evaluated the humoral immune response against SARS-CoV-2 in subjects during the first and second waves of the pandemic in Morocco.

Methods: We assessed humoral immune response in samples from 94 seropositive individuals in the first wave (February to August 2020) and 596 seropositive individuals in the second wave (December 2020 to January 2021). Plasma samples were collected from volunteer blood donors and their levels of serum IgG to SARS-CoV-2 nucleoprotein (NP) were determined using architect SARS-CoV-2 IgG chemiluminescence microparticle immunoassay.

Results: Our results revealed an increase of humoral immunity during the second wave than first wave ( $3.897 \pm 0.079$ vs. $2.842 \pm 0.153$, respectively, $p<0.0001)$. Notably, we found an age-related gradient in antibody level, with higher antibody index in subjects at 45 years old and above $(p=0.01451)$. However, no significant difference was found according to gender $(p=0.8629)$.

Conclusions: Our data highlighted an important issue regarding antibody-mediated immune response against SARS-CoV-2 infection during the second wave and this issue might have arisen due to the dynamics of different strains circulating during the progress of the pandemic.

\section{Introduction}

Severe acute respiratory syndrome coronavirus 2 (SARS-CoV-2) -the causative agent of the ongoing pandemic of coronavirus disease 2019 (COVID-19)-emerged one year ago ${ }^{1}$, and is now posing further challenges in many countries worldwide as new variants are identified. These variants include those identified in the United Kingdom (UK) (variant 20I/501Y.V1, VOC 202012/01, or B.1.1.7), South Africa (variant 20H/501Y.V2 or B.1.351), and Brazil (variant P.1), and have raised many questions around severity of disease, increased risk of death, vaccine efficacy, and the speed at which COVID-19 may proliferate ${ }^{2-8}$.

The North African kingdom of Morocco has adopted a range of measures to combat the COVID-19 pandemic, with surveillance for new variants including diagnostic and screening measures with nucleic acid amplification tests, antigen-based tests, serologic assays and genome sequencing among the approaches that have been applied. Previously, Morocco appeared to achieve a degree of control against the disease since the country's Ministry of Health first reported a case, on March 2nd, 2020. The $5,443,945$ tests carried out in Morocco as of March 28th, 2021 yielded a total of 494,659 positive results, with 8,798 deaths and 482,352 patients recorded as recovering from COVID-19. In January 28th, 2021, the kingdom launched the national vaccination campaign and as of March 28th, 2021, 4,302,183 persons 
received their first dose and 3,332,292 second dose of a COVID-19 vaccine. As with many other countries, Morocco appears to have experienced waves of infection, with the first wave regarded as commencing with the first reported case in March 2020, and the second wave around September 2020, just weeks after the traditional Eid al-Adha festivities, which are marked by social and religious gatherings. The two waves are separated by a trough in daily recorded cases (Fig. 1). However, on January 18th, 2021, Morocco's health ministry confirmed its first imported case of the UK variant, which was detected in the northern port of Tangier in a Moroccan national returning from Ireland via Marseille.

With recently described variants in SARS-CoV-2, and characterizing antibody (Ab)-mediated immune response dynamics of SARS-CoV-2 is fundamental to understand COVID-19 epidemiology, the path to protection against SARS-CoV-2 infection or re-infection, and for effective vaccine development ${ }^{9}$. Data of this nature are not yet available for SARS-CoV-2 infection. To investigate the humoral immune response against SARS-CoV-2 infection in the first and second waves in Morocco, we subjected blood samples from donors who recovered from COVID-19 during the first wave, or seroconverted during the second wave to a comparative evaluation.

\section{Matarials And Methods}

\section{Study participants}

This is an observational study in healthy volunteers. We enrolled two groups of donors in two different periods, between 14th of February and 7th of August 2020, for the first wave), or 23th of December 2020 through January 8, 2021 (for the second wave). Blood donors satisfying the donation eligibility criteria set by the Moroccan Ministry of Health and testing positive for SARS-CoV-2 could be included in the study. Individuals underwent a health screening procedure before blood donation. After enrolment, participants were grouped into three age categories: $18-25$ years, $26-45$ years and more than 45 years. The samples were collected from Regional Blood Transfusion Center in Casablanca and Blood Transfusion Center in El Jadida. The study protocol was in accordance with the Helsinki declaration and received an approval from the ethics Committees of the Faculty of Medicine of Rabat $\left(\mathrm{N}^{\circ} 17 / 20\right)$ and Ibn Rochd University Hospital ( $\left.{ }^{\circ} 02 / 21\right)$ and written informed consent was obtained from each participant.

\section{Serological testing}

Serologic tests to ascertain the presence of the antibody against SARS-CoV-2 (IgG) were conducted at the Viral Hepatitis Laboratory of the Pasteur Institute of Morocco. We used Architect ${ }^{\text {TM }}$ SARS-CoV-2 IgG chemiluminescence microparticle immunoassay (CMIA) (Abbott Park, Illinois, USA) to detect serum IgG Abs directed against the nucleocapsid protein of SARS-CoV-2. Interpretation of results was based on manufacturer's criteria: negative when the cut-off index $(\mathrm{S} / \mathrm{C})<1.4$ and positive when the cut-off index $(\mathrm{S} / \mathrm{C}) \geq 1.4$.

\section{Statistical Analysis}


Data are presented as mean values (with corresponding standard errors of mean). Two-tailed Student's ttest or one-way ANOVA were used to analyze the differences between groups. Correlations between antibody index and age were assessed using Pearson's correlation coefficient. Statistical analyses were performed using GraphPad PRISM version 6.0e (GraphPad Software, San Diego, CA, USA).

\section{Results}

Among 14,000 blood donors screened in the first wave, 94 individuals who tested positive for SARS-CoV2- $\lg G$, and were included in the study population as the first-wave cohort. Among 1,986 blood donors in the second wave, 596 individuals were positive for SARS-CoV-2- IgG, and were included in the study population as the second-wave cohort.

The 94 COVID-19-recovered subjects in the first wave had a mean age of $38 \pm 10$ years and sex ratio (male/female) of 1. The 596 COVID-19- recovered subjects in the second wave had a mean age of $36 \pm 10$ years and sex ratio (male/female) of 2.16. No significant difference was observed regarding age between these cohorts $(p=0.2325)$. However, significant difference was observed regarding sex between these cohorts $(p=0.0002)$. Antibody index analyses revealed that the second-wave cohort had an elevated humoral immunity relative to the first-wave cohort ( $3.897 \pm 0.079$ vs. $2.842 \pm 0.153, p<0.0001$, respectively) (Fig. 2A).

We tested for associations of age and sex with antibody index among subjects in the second wave cohort. Notably, we found an age-related gradient in antibody levels $(p=0.01451)$ (Fig. 2B \& Fig. 2C). Interestingly, SARS-CoV-2 antibody index was higher in individuals aged 45 years or above (Fig. 2B). However, no significant difference was observed between men and woman regarding antibody index $(p=$ 0.8629) (Fig. 2D).

\section{Discussion}

Herein, we report a comparative evaluation of humoral immune response in SARS-CoV-2-IgG positive blood donors covering two waves of infection over a period of more than 11 months. We found that antibody response was greater in the second than the first wave. However, we are unable to assess the functionality of antibodies during the first and second waves due to lack of virus neutralization assay. However, the $\mathrm{N}$ protein is predicted to serve as a surrogate marker for the $\mathrm{S}$ protein-neutralizing antibody complex ${ }^{10}$. While all viral proteins are vulnerable to mutations that could impact diagnostic test performance, the $S$ protein is under more selective pressure ${ }^{2}$.

This atypical humoral immunity is particularly relevant in Morocco, where the second wave commenced around September 2020 and was probably linked to multiple distinct variants having accumulated mutations that differed from those in variants circulating during the first wave. Indeed, the SARS-CoV-2 strains circulating during the early epidemic phase in Morocco are different to those circulating in the second wave. Analysis of Moroccan SARS-CoV-2 sequences from the first wave clustered SARS-CoV-2 
strains in clades G, B11, and A2 with the presence of amino acid change at D614G, which resembles strains seen in Asia, Europe, North America, Australia, and Africa, this indicating multiple SARS-CoV-2 introductions into Morocco ${ }^{11-13}$. Similarities are also evident with European strains seen at the beginning of the pandemic, and SARS-CoV-2 strains isolated in America and Africa later in the pandemic ${ }^{14}$. In addition, in Morocco, SARS-CoV-2 strains identified during December 2020 revealed an increased number of mutations relative to those identified in the first wave ${ }^{15,16}$. This pattern appears to be in line with previous reports showing that viral genomes from the first and second waves belong to different clades/lineages ${ }^{17-19}$. Moreover, several recent reports of reinfection, as indicated by viral sequence differences, clinical data and data on potential exposure, have raised critical questions about whether and how well a first infection protects against reinfection ${ }^{9,17-20}$. In addition, a relationship between a humoral immune response to SARS-CoV-2 infection and protection against reinfection with the identical SARS-CoV-2 strain has been shown in rhesus macaques, but has yet to be established in humans ${ }^{21}$. However, with the emergence of new variants more studies are needed to determine whether the SARSCoV-2 variants reflect antigenic differences between strains circulating in the first and second waves, or waning immunity against SARS-CoV-2 ${ }^{17}$.

In this study, older age was associated with greater IgG responses to SARS-CoV-2 but gender did not affect this association. Previous studies showed that older age is associated with greater antibody production and that males consistently had greater antibodies levels ${ }^{22,23}$.

In conclusion, our data revealed an important issue regarding antibody-mediated immune response against SARS-CoV-2 infection during the second wave. This issue may have arisen due to the dynamics of differences between circulating strains as the pandemic progressed and/or between specific human genetic variants that contribute to inter-individual variability in serum immunoglobulin levels ${ }^{24}$.

There are limitations associated with this study. The antibody response induced by COVID-19 tends to be linked to the severity of the disease. However, the disease severity could not be determined in the recovering subjects. The study was also limited by the lack of assessment of neutralizing antibody levels. Further investigations are warranted to determine whether common or divergent factors are driving this drift in humoral immunity during the COVID-19 pandemic.

\section{Declarations}

\section{Declaration of Competing Interest}

All authors declare that there are no conflicts of interests.

\section{Funding}

This study was supported by Moroccan Ministry of Health and research grant from COVID19 Taskforce (Institut Pasteur, Paris) 


\section{Conflicts of interest}

None

\section{Availability of data and material}

The datasets used and analyzed during the current study are available from the corresponding author on reasonable request.

\section{Code availability}

Not applicable

\section{Ethics approval and consent to participate}

The study protocol was in accordance with the Helsinki declaration and received an approval from the ethics Committees of the Faculty of Medicine of Rabat $\left(\mathrm{N}^{\circ} 17 / 20\right)$ and Ibn Rochd University Hospital $\left(\mathrm{N}^{\circ} 02 / 21\right)$ and written informed consent was obtained from each participant.

\section{Author Contribution}

S.E, S.B, A.M, N.R, H.M, and S.R participated in the conception and design of the study. N.M and A.Z collected samples and data. I.A, C.Z, A.H, S.H, K.A, O.L, and A.A conducted the serological assay. S.E, S.B, A.M, H.M, and S.R participated in acquisition and interpretation of the data. S.E and M.E analyzed the data. S.E wrote the manuscript with assistance from all coauthors and approved the version of paper.

\section{Consent for publication}

All authors approved the final version for publication.

\section{Acknowledgments}

We acknowledge the contributions of other clinical and technical staff of the Regional Blood Transfusion Centers in Casablanca and El Jadida and from Ministry of Health.

\section{References}

1 Wang, C., Horby, P. W., Hayden, F. G. \& Gao, G. F. A novel coronavirus outbreak of global health concern. Lancet 395, 470-473, doi:10.1016/S0140-6736(20)30185-9 (2020).

2 Korber, B. et al. Tracking Changes in SARS-CoV-2 Spike: Evidence that D614G Increases Infectivity of the COVID-19 Virus. Cell 182, 812-827.e819, doi:10.1016/j.cell.2020.06.043 (2020).

3 Rambaut, A. et al. Preliminary genomic characterisation of an emergent SARS-CoV-2 lineage in the UK defined by a novel set of spike mutations. Virological.org. Posted December 16, 2020. Accessed January 
19, 2021. https://virological. org/t/preliminary-genomic-characterisation-of-an-emergent-sars-cov-2lineage-in-the-uk-defined- by-a-novel-set-of-spike-mutations/563. (2020).

4 Tegally, H. et al. Emergence and rapid spread of a new severe acute respiratory syndrome-related coronavirus 2 (SARS-CoV-2) lineage with multiple spike mutations in South Africa.Preprint at medRxiv. doi:https://doi.org/10.1101/2020.12.21.20248640 (2020).

5 Kirby, T. New variant of SARS-CoV-2 in UK causes surge of COVID-19. Lancet Respir Med S2213-2600, 00005-00009, doi:10.1016/S2213-2600(21)00005-9 (2021).

6 Callaway, E. Could new COVID variants undermine vaccines? Labs scramble to find out. Nature $\mathbf{5 8 9}$, 177-178, doi:10.1038/d41586-021-00031-0 (2021).

7 Xie, X. et al. Neutralization of N501Y mutant SARS-CoV-2 by BNT162b2 vaccine-elicited sera. bioRxiv. . doi:10.1101/2021.01.07.425740 (2021).

8 Resende, P. C. et al. Spike E484K mutation in the first SARS-CoV-2 reinfection case confirmed in Brazil, 2020. Virological.org. Posted January 10, 2021 Accessed January 20, 2021.

https://virological.org/t/spike-e484k-mutation-in-the-first-sars-cov-2-reinfection-case-confirmed-in-brazil2020/584. (2021).

9 Overbaugh, J. Understanding protection from SARS-CoV-2 by studying reinfection. Nat Med 26, 16801681, doi:10.1038/s41591-020-1121-z. (2020).

10 Batra, R. et al. A comparative evaluation between the Abbott Panbio ${ }^{\text {TM }}$ COVID-19 IgG/IgM rapid test device and Abbott Architect ${ }^{\text {TM }}$ SARS CoV-2 IgG assay. J Clin Virol 132, 104645, doi:10.1016/j.jcv.2020.104645 (2020).

11 Lemriss, S. et al. Complete Genome Sequence of a 2019 Novel Coronavirus (SARS-CoV-2) Strain Causing a COVID-19 Case in Morocco. Microbiol Resour Announc 9, e00633-00620, doi:10.1128/MRA.00633-20 (2020).

12 Jouali, F. et al. SARS-CoV-2 Genome Sequence from Morocco, Obtained Using lon AmpliSeq Technology. Microbiol Resour Announc 9, e00690-00620, doi:10.1128/MRA.00690-20 (2020).

13 Laamarti, M. et al. Genome Sequences of Six SARS-CoV-2 Strains Isolated in Morocco, Obtained Using Oxford Nanopore MinION Technology. Microbiol Resour Announc 9, :e00767-00720, doi:10.1128/MRA.00767-20 (2020).

14 Song, S. et al. The global landscape of SARS-CoV-2 genomes, variants, and haplotypes in 2019nCoVR. bioRxiv, 2020.2008.2030.273235, doi:10.1101/2020.08.30.273235 (2020).

15 Fournier, P. E. et al. Emergence and outcome of the SARS-CoV-2 "Marseille-4" variant. Preprint IHU doi: https://doi.org/10.35081/xcrm-6t77. (2021). 
16 Colson, P. et al. Introduction into the Marseille geographical area of a mild SARS-CoV-2 variant originating from sub-Saharan Africa: an investigational study. Travel Med Infect Dis 101980, doi:10.1016/j.tmaid.2021.101980 (2021).

17 To, K. K. et al. Serum antibody profile of a patient with COVID-19 reinfection. Clin Infect Dis ciaa1368, doi:10.1093/cid/ciaa1368 (2020).

18 Colson, P., Finaud, M., Levy, N., Lagier, J. C. \& Raoult, D. Evidence of SARS-CoV-2 re-infection with a different genotype. J Infect S0163-4453, 30706-30704, doi:10.1016/j.jinf.2020.11.011 (2020).

19 Colson, P. et al. Dramatic increase in the SARS-CoV-2 mutation rate and low mortality rate during the second epidemic in summer in Marseille. Preprint IHU 2020;doi:https://doi.org/10.35088/68c3-ew82 . (2020).

20 Tillett, R. L. et al. Genomic evidence for reinfection with SARS-CoV-2: a case study. Lancet Infect Dis21, 52-58, doi:10.1016/S1473-3099(20)30764-7 (2021).

21 Deng, W. et al. Primary exposure to SARS-CoV-2 protects against reinfection in rhesus macaques. Science 369, 818-823, doi:10.1126/science.abc5343 (2020).

22 Klein, S. L. et al. Sex, age, and hospitalization drive antibody responses in a COVID-19 convalescent plasma donor population. J Clin Invest 130, 6141-6150, doi:10.1172/JCI142004 (2020).

23 Zhang, B. et al. Immune Phenotyping Based on the Neutrophil-to-Lymphocyte Ratio and IgG Level Predicts Disease Severity and Outcome for Patients With COVID-19. Front Mol BioscI7, 157, doi:10.3389/fmolb.2020.00157 (2020).

24 Scepanovic, P. et al. Human genetic variants and age are the strongest predictors of humoral immune responses to common pathogens and vaccines. Genome Med10, 59, doi:10.1186/s13073-018-0568-8 (2018).

\section{Figures}

A

Montly COVID-19 Cases in Morocco

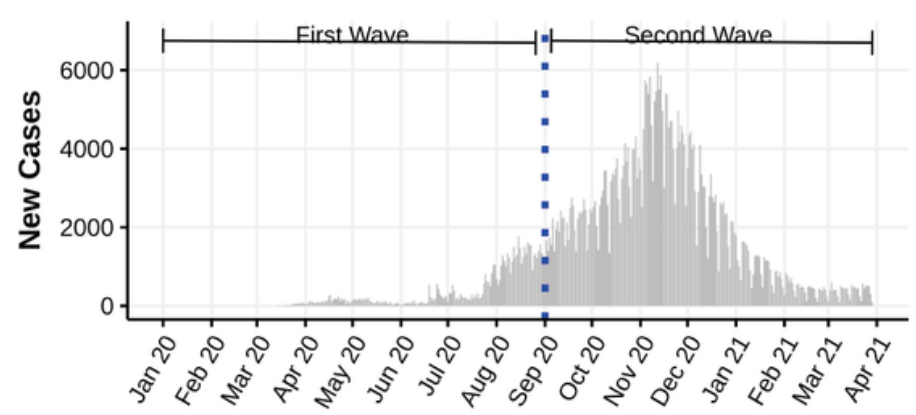

Date of Report
B Montly CoVID-19 Deaths in Morocco

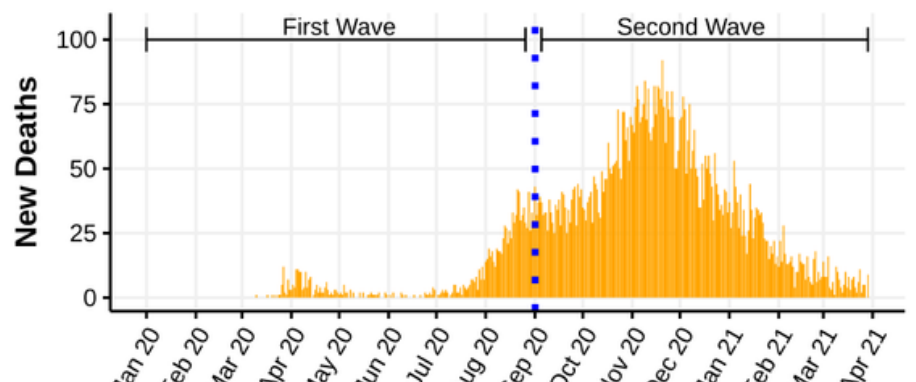

Date of Report 
Figure 1

SARS-CoV-2 epidemiological dynamics in Morocco. A). The epidemic curve of new confirmed COVID-19 cases. B) Daily confirmed COVID-19 deaths.
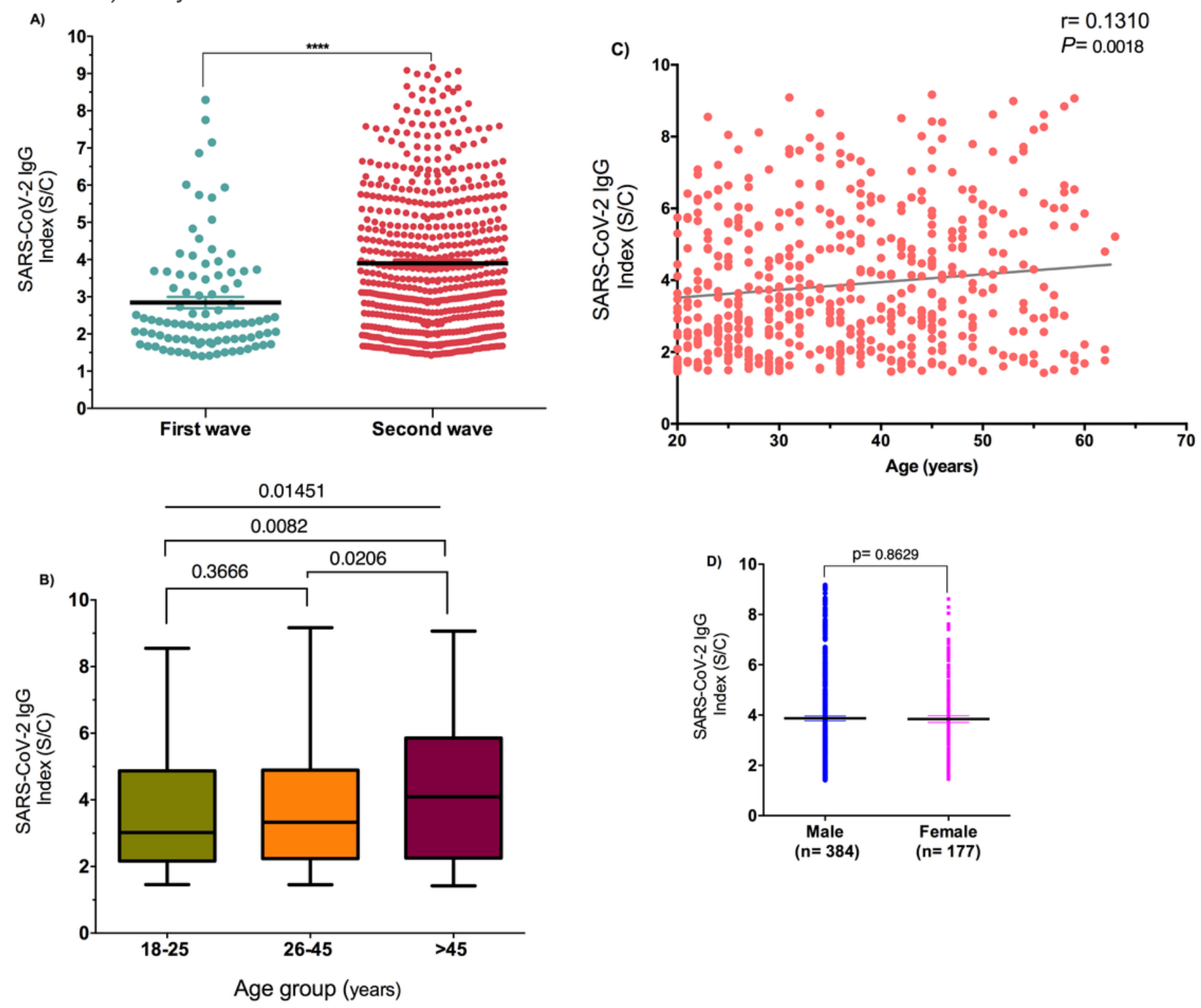

Figure 2

Antibody index of seropositive subjects. A) Comparison between first and second waves. Data are presented as mean and standard error of mean (SEM). B) Box and whiskers plot of antibody index with regard to age. The line crossing the box represents the mean. The whiskers extend to the minimal and maximal observed in each cohort. C) Age is associated with antibody responses to SARS-CoV-2. D) The graphic shows aligned dot plot of antibody index according to gender. Data are presented as mean \pm SEM. Data were compared using Student's two-tailed t-test or one-way ANOVA. 